\title{
Tooth wear in patients treated with HIV anti-retroviral therapy
}

\author{
Harjit Singh Sehgal ${ }^{1 *}$, Richie Kohli ${ }^{2}$, Edward Pham ${ }^{3}$, Grace E. Beck ${ }^{4}$ and Jay R. Anderson ${ }^{5}$
}

\begin{abstract}
Background: The objective of this study was to elucidate the relationship between HIV anti-retroviral therapy and tooth wear.

Methods: Assessment of tooth wear was conducted both with a survey questionnaire and clinical assessment at Russell Street Dental Clinic in Portland, Oregon. The survey questionnaire comprised of questions on study participant's gender, age, HIV status, current medications, awareness of tooth grinding or clenching, jaw soreness, tooth or gum soreness, and frequency of headaches. For the clinical evaluation, a dental provider recorded the degree of wear on each tooth using a scale of $0-3$. An individual tooth-wear index was used to rank patients with regard to incisal and occlusal wear. Data analysis included descriptive analysis, tests of association and regression analysis using SPSS V.24.
\end{abstract}

Results: The study sample involved 93 patients ( $H I V+v e=60, H I V-v e=33$ ) with age range of 20-90 yrs. (mean $=49$ yrs., s.d = 13.3). 92 and $67 \%$ participants of the HIV + ve and HIV-ve groups, respectively, presented with tooth wear. The mean tooth wear index was higher in HIV + ve patients than HIV-ve patients (8.2 vs. 7.8), however, this difference was not statistically significant $(p>0.05)$. A significant, positive correlation was found between HIV presence and tooth wear index, after accounting for age $(B=0.71, p<0.05)$. The number of years on anti-retroviral therapy alone was positively correlated with tooth wear index $\left(R^{2}=0.116, p<0.05\right)$. After controlling for age, years of anti-retroviral therapy use was positively correlated with tooth wear index $(B=0.047, p>0.05)$.

Conclusions: The findings from this study suggest that HIV + ve patients, who are on anti-retroviral therapy have significant tooth wear, although more studies with larger sample size are needed to confirm this. There is a critical need to initiate a dialogue with medical providers about tooth wear as a possible side effect of antiretroviral therapy and to introduce appropriate preventive measures.

Keywords: Tooth wear, Bruxism, Community dentistry, Dental, Anti-retroviral therapy

\section{Background}

People living with human immunodeficiency virus (HIV) and acquired immunodeficiency syndrome (AIDS) (PLWHA) face tremendous problems regarding their oral health. PLWHA have an increased incidence of poor oral health, which further exacerbates their other medical conditions. PLWHA have reported high unmet oral health needs and low utilization of oral health services $[8,19]$. Furthermore, PLWHA face many barriers to acquiring oral health care, including lack of dental

\footnotetext{
* Correspondence: sehgal@ohsu.edu

${ }^{1}$ Department of Periodontology, School of Dentistry, Oregon Health \& Science University, 5N034 SDPERI, 2730 SW Moody Ave, Portland, Oregon 97201-5042, USA

Full list of author information is available at the end of the article
}

insurance, limited financial resources [8], shrinking adult dental Medicaid services [19], and perceived stigma within health care systems $[28,29]$.

Clinical observation has suggested that many PLWHA are diagnosed with bruxism. Bruxism is characterized by the involuntary clenching or grinding of the teeth especially during sleep and can cause severe oral health problems, including the destruction of tooth structure, temporomandibular joint dysfunction, myofascial pain, and severe sleep disturbances [13, 14, 17, 30]. Bruxism has been reported to occur about two times more frequently in PLWHA than in the general population. In a systematic review of 26 papers, researchers found two articles stating bruxism has a prevalence of up to $31 \%$ of

(c) The Author(s). 2019 Open Access This article is distributed under the terms of the Creative Commons Attribution 4.0 International License (http://creativecommons.org/licenses/by/4.0/), which permits unrestricted use, distribution, and reproduction in any medium, provided you give appropriate credit to the original author(s) and the source, provide a link to the Creative Commons license, and indicate if changes were made. The Creative Commons Public Domain Dedication waiver (http://creativecommons.org/publicdomain/zero/1.0/) applies to the data made available in this article, unless otherwise stated. 
adults [18]. In a study conducted by Juvino et al. in 2017, 64\% of HIV/AIDS participants presented with bruxism.

Not only are AIDS patients susceptible to numerous oral and systemic ailments, but also these individuals are faced with psychological challenges. PLWHA are more likely to develop depression and anxiety than the general population ([2]; Sun, Wu, Ou, Lu, \& Wang, 2014). Both depression and anxiety affect a person's quality of life [5, 34] as well as their ability to follow the treatment regimen for HIV and attend to their oral self-care [3, 15, 25]. The presence of psychological disorders is known to result in various oral health problems, such as bruxism $[6,21]$ and tooth wear [23]. Several researchers found bruxers to have increased incidence of anxiety than nonbruxers [12, 16]. While currently available depression and anxiety treatments are generally well tolerated and safe, a class of medications used to treat depression, selective serotonin reuptake inhibitors (SSRIs), increases teeth grinding and therefore bruxism [26]. The combination of HIV, medications for HIV treatment, and the impact of SSRI may lead to progressively severe dental attrition.

The antiretroviral medications that PLWHA depend on have negative effects on their oral health. Many oral health issues experienced by PLWHA are related to the side effects of the medications themselves, including xerostomia (or dry mouth) which is a significant complication of HIV and/or side effect of ART medications [27]. In addition, antiretroviral therapy can have various side effects including mood and sleeping disturbances [1]. The combination of insomnia and medication side effects increases the likelihood of clenching and grinding of PLWHA patients. HIV drugs in combination with other medications that can cause dry mouth [33], clenching and grinding (A.K. Johansson, Omar, Carlsson, \& A. Johansson, 2012) leave teeth surfaces vulnerable to both mechanical wear and chemical wear [24, 31].

Antiretroviral therapy can have various other side effects including weight gain, weight loss, bone and bleeding problems, as well as an increased risk of heart disease, kidney and/or liver problems (Johansson, 2012). However, not many studies exist regarding oral health and parafunctional habits associated with medications used to treat AIDS. Those studies that do exist examine the oral health pathologies experienced as a result of opportunistic infections, rather than the therapeutic regimens [20]. Due to the lack of research, this topic is not well understood and little is known about the effects of antiretroviral therapy on oral health. The present study is among the few in the literature and the first in Oregon to establish an association between tooth wear and the antiretroviral medications taken by HIV positive patients.

\section{Methods}

This study protocol was approved by the Oregon Health \& Science University Institutional Review Board (IRB00010706).

\section{Study design}

This is a cross-sectional study that utilizes survey and clinical assessment of dentition among HIV positive patients on antiretroviral therapy. Participants were recruited from the Russell Street Clinic, a walk-in, community dental clinic in Portland, Oregon. Patients were screened by survey. Surveys asked participants to self-report whether they have symptoms of bruxism. Additional subject characteristics were documented. A dentist conducted clinical evaluations. Following the study, informational brochures regarding the side effects of HIV treatment on oral health were developed and distributed among patients at the clinic.

\section{Sample}

At the Russell Street Clinic, patients were screened based on eligibility criteria as follows: at least 18 years of age, not pregnant, have at least 25 natural permanent teeth, HIV positive (HIV + ve) and on highly active antiretroviral therapy (HAART), or HIV negative (HIV-ve). If the patient was missing more than seven of his or her natural teeth, they were excluded from the study. Consent was provided at time of arrival to clinic. Spanishspeaking patients who met study criteria completed the surveys that were translated to their native language. In person interviews were conducted in either English or Spanish to screen participants.

\section{Data collection}

Data was collected over the time span of one month (29 days) from June to July 2014. Upon receiving consent, patients were administered a survey in either English or Spanish. The survey asked participants to report various demographic and health information as reported in Table 1 and Table 2. In addition, participants were asked

Table 1 Demographics $(N=93)$

\begin{tabular}{lll}
\hline Demographic Variables & $\begin{array}{l}\text { HIV +ve } \\
(n=60)\end{array}$ & $\begin{array}{l}\text { HIV-ve } \\
(n=33)\end{array}$ \\
\hline Gender & $\mathrm{n}(\%)$ & $\mathrm{n}(\%)$ \\
$\quad$ Male & $56(93)$ & $16(48)$ \\
$\quad$ Female & $3(5)$ & $17(52)$ \\
Age [mean (SD)] & $50(10)$ & $48(18)$ \\
Smoking & $23(38)$ & $7(21)$ \\
Alcohol Use (> 7 bev./wk) & $9(15)$ & $7(21)$ \\
Reported Recreational Drug Use & $9(15)$ & $2(6)$ \\
\hline
\end{tabular}


Table 2 Clinical Variables Among HIV+ and HIV- Participants

\begin{tabular}{llll}
\hline Clinical Variables & $\begin{array}{l}\text { HIV }+ \text { ve } \\
(n=60) \\
n(\%)\end{array}$ & $\begin{array}{l}\text { HIV-ve } \\
(n=33)\end{array}$ & $p^{*}(\%)$ \\
\hline Temporomandibular Joint Disorder Diagnosis & $6(10)$ & $1(3)$ & 0.223 \\
Bite Guard Therapy & $7(12)$ & $1(3)$ & 0.155 \\
Clenching/Grinding: & & & \\
Day & $29(48)$ & $8(24)$ & 0.023 \\
Night & $23(38)$ & $5(15)$ & 0.020 \\
Teeth/Gum Soreness & $25(42)$ & $5(15)$ & 0.009 \\
Jaw Soreness & $21(35)$ & $5(15)$ & 0.041 \\
Headaches & $15(25)$ & $4(12)$ & 0.141 \\
Psychiatric Disorder & $30(50)$ & $7(21)$ & 0.007 \\
Tooth Wear & $55(92)$ & $22(67)$ & 0.002 \\
\hline * p-value from Chi-squared test & & &
\end{tabular}

to self-report symptoms associated with bruxism, including teeth grinding or clenching during the day or night, morning jaw soreness, tooth and/or gum soreness, and presence and frequency of headaches. HIV positive participants were asked to report how long they had been treated with HAART. Following completion of the survey, patients proceeded with their regular dental care visit, and clinicians utilized an assessment tool developed based on the study conducted by Ekfeldt, Hugoson, Bergendal, \& Helkimo in 1990.

\section{Clinical exam}

The dentist first recorded the total number of teeth present and then the severity of enamel erosion on each tooth was recorded using the assessment tool. Level of enamel erosion was documented: the number of teeth that had no obvious wear of enamel (Level 0 Erosion); the number of teeth that had wear through the enamel to the dentin in single spots (Level 1 Erosion); the number of teeth that had wear of the dentin up to one-third of the crown height (Level 2 Erosion); and the number of teeth that had wear of the dentin up to more than one-third of the crown height, excessive wear of tooth restorative material or dental material in the crown and bridgework (Level 3 Erosion).

Participants were sorted into three age cohorts: $18-40$, 41-65, and 66 and older. Separation into age cohorts helped control for the effect of age on natural enamel erosion and tooth wear. The proportion of participants with teeth that had Level 1, Level 2, and Level 3 enamel erosion was determined. Within each age cohort, the proportion of Level 1, 2, and 3 enamel erosion in HIV patients treated with antiretroviral therapy was compared to a control group of non-HIV patients who were not treated with antiretroviral therapy.
Individual tooth wear index was computed via methods founded by Ekfeldt et al. [7]. This tooth wear index is well reported in the literature as a reliable and clinically valid formula for calculating the degree of tooth wear (Koyano et al., 1990; [4]). This index has been shown to be significantly correlated with bruxism [9]. The following tool allows for indexes to be calculated without influence by amount of tooth loss:

$$
I_{A}=\left(10 \times G_{1}+30 \times G_{2}+100 \times G_{3}\right) /\left(G_{0}+G_{1}+G_{2}+G_{3}\right)
$$

where $I_{A}$ is individual tooth-wear index and $G_{0}, G_{1}, G_{2}$ and $G_{3}$ are the number of teeth with scores of $0,1,2$ and 3 , respectively.

\section{Data analysis}

Within each age cohort, we used STATA to conduct an ANOVA with age group as a factor and correct for potential confounders, including anxiety, depression, autism, schizophrenia, ADHD (attention deficit hyperactivity disorder), OCD (obsessive compulsive disorder), bipolar disorder or other psychiatric and psychological disorders. As an observational study, a sample size calculation is not applicable. In addition, descriptive statistics and tests of association (including t-tests, various cross-tabulations, Pearson Chi-Square tests) were conducted using SPSS version 24.

\section{Results}

We examined 104 participants in total. A total of 11 participants did not receive wear scores due to not meeting criteria (7 HIV + ve, 4 HIV-ve) and were excluded from all analyses, resulting in a final sample size of 93, of whom 60 were HIV positive (65\%) and 33 were HIV negative (35\%). A majority of HIV + ve participants were male (93\%). A greater proportion of HIV + ve subjects compared to HIV-ve subjects reported the use of tobacco cigarettes and recreational drugs. Those with HIV also had a greater mean age than those without HIV. However, this age difference was 2 years (50 vs. 48 , respectively) (Table 1). All 60 HIV + ve subjects were on at least one antiretroviral medication.

Additional clinical variables were measured between HIV + ve and HIV-ve, including presence of temporomandibular disorder (TMD), use of night guard therapy, clenching/grinding during day and at night, teeth/gum soreness, jaw soreness, headaches, psychiatric disorder, and tooth wear (Table 2). A significantly greater proportion of HIV + ve subjects clenched during the day, clenched during night, experienced teeth/gum and jaw soreness, tooth wear, as well as some form of psychiatric disorder when compared to HIV-ve subjects $(p<0.05)$. Although statistically insignificant, a greater proportion 
of HIV + ve individuals than HIV-ve individuals reported TMD, bite guard therapy and headaches.

The presence of tooth wear and mean tooth wear index was calculated and compared between HIV + ve and HIV-ve groups. As seen in Tables 2, 92 and 67\% participants of the HIV + ve and HIV-ve groups, respectively, presented with tooth wear (Level 1, 2, or 3). The mean tooth wear index of HIV + ve subjects was higher than the non-HIV infected subjects, though this finding is statistically insignificant (Table 3 ). However, this demonstrates that among the HIV + ve subjects, there was an average greater number of teeth that presented with tooth wear, or an average lower total number of teeth with wear but with a greater degree of tooth wear on each tooth. Mean tooth wear was also determined within the HIV + ve group and compared between the years of antiretroviral therapy use (1-5 years, $6-10$ years, $11-15$ years, $16-20$ years, $21-25$ years, and $26-30$ years). As years of antiretroviral therapy use increased, there was an increase in mean tooth wear (Fig. 1).

All participants were categorized by their characteristics and mean tooth wear index was calculated for each characteristic. Multiple independent samples t-tests produced the following results (Table 3). Those who reported clenching during the day presented with nearly twice as much tooth wear than those who reported no clenching $(p<0.05)$. Male subjects tended to have nearly three times as much tooth wear as female subjects $(p<$ 0.01 ). Interestingly, those who reported drinking 7 or more alcoholic beverages per week presented with half as much tooth wear than those who consumed less alcohol $(\mathrm{p}<0.05)$. Though statistically insignificant, other notable findings include a larger mean tooth wear index among those reporting clenching at night, bite guard therapy, headaches, teeth/gum soreness, and retroviral therapy.

\section{Regression analysis}

Following linear regression analysis, the results of Table 4 were produced. HIV + ve status is positively correlated with tooth wear index* $\left(\mathrm{R}^{2}=0.06, p\right.$ value $=0.017$ ). Both HIV + ve status and age are positively correlated to tooth wear index $\left(R^{2}=0.13\right.$, $\mathrm{p}$ value $=0.002$ ). After accounting for age, a significant, positive correlation was found between HIV presence and tooth wear index $(\mathrm{B}=0.71, p=0.025)$. Age was positively correlated with years of anti-retroviral therapy $\left(n=58, \mathrm{R}^{2}=0.27, p<0.001\right)$. This decrease in sample size from 60 was due to those subjects who did not report the duration of anti-retroviral therapy use. Age alone is also positively correlated to tooth wear index $\left(R^{2}=0.08\right.$, p-value $\left.=0.006\right)$. The number of years on anti-retroviral therapy alone is positively correlated with tooth wear index $\left(\mathrm{R}^{2}=0.116, p=\right.$
Table 3 Mean Tooth Wear Index by Presence or Absence of Characteristics ( $\mathrm{N}=93$ )

\begin{tabular}{|c|c|c|}
\hline Characteristics & Tooth Wear Index (s.d.) & $\begin{array}{l}p \text {-value (two tailed } \\
\text { t-test unequal variances) }\end{array}$ \\
\hline \multicolumn{3}{|l|}{ HIV } \\
\hline+ & $8.2(10.0)$ & 0.216 \\
\hline- & $7.8(8.3)$ & \\
\hline \multicolumn{3}{|l|}{ Smoking } \\
\hline+ & $6.5(6.6)$ & 0.530 \\
\hline- & $7.7(10.8)$ & \\
\hline \multicolumn{3}{|c|}{ Temporomandibular Joint Disorder } \\
\hline+ & $6.1(4.2)$ & 0.506 \\
\hline- & $7.4(9.8)$ & \\
\hline \multicolumn{3}{|c|}{ Bite Guard Therapy } \\
\hline+ & $15.8(22.8)$ & 0.291 \\
\hline- & $6.5(6.9)$ & \\
\hline \multicolumn{3}{|l|}{ Clenching-Day } \\
\hline+ & $10.6(12.8)$ & $0.018^{*}$ \\
\hline- & $5.2(5.5)$ & \\
\hline \multicolumn{3}{|l|}{ Clenching-Night } \\
\hline+ & $10.6(13.9)$ & 0.101 \\
\hline- & $6.0(6.4)$ & \\
\hline \multicolumn{3}{|c|}{ Psychiatric Disorder } \\
\hline+ & $8.5(11.5)$ & 0.394 \\
\hline- & $6.6(7.9)$ & \\
\hline \multicolumn{3}{|c|}{ Anti-Retroviral Therapy } \\
\hline+ & $8.2(10.0)$ & 0.216 \\
\hline- & $5.8(8.3)$ & \\
\hline \multicolumn{3}{|l|}{ Sex } \\
\hline Male & $8.4(10.2)$ & $0.005^{*}$ \\
\hline Female & $3.5(5.4)$ & \\
\hline \multicolumn{3}{|l|}{ Jaw Soreness } \\
\hline+ & $8.6(7.1)$ & 0.348 \\
\hline- & $6.8(10.3)$ & \\
\hline \multicolumn{3}{|l|}{ Headaches } \\
\hline+ & $10.3(8.2)$ & 0.098 \\
\hline- & $6.6(9.7)$ & \\
\hline \multicolumn{3}{|c|}{ Teeth/Gum Soreness } \\
\hline+ & 10.0 (13.3) & 0.130 \\
\hline- & $6.0(6.7)$ & \\
\hline \multicolumn{3}{|c|}{7 or more alcoholic beverage per week } \\
\hline+ & $4.4(4.1)$ & $0.024^{*}$ \\
\hline- & $8.0(10.1)$ & \\
\hline
\end{tabular}

${ }^{*} p$-value $<0.05$

0.009). Together, age and years of anti-retroviral therapy explained about $13 \%$ of the variability in tooth wear $\left(R^{2}=0.13, p=0.021\right)$. 


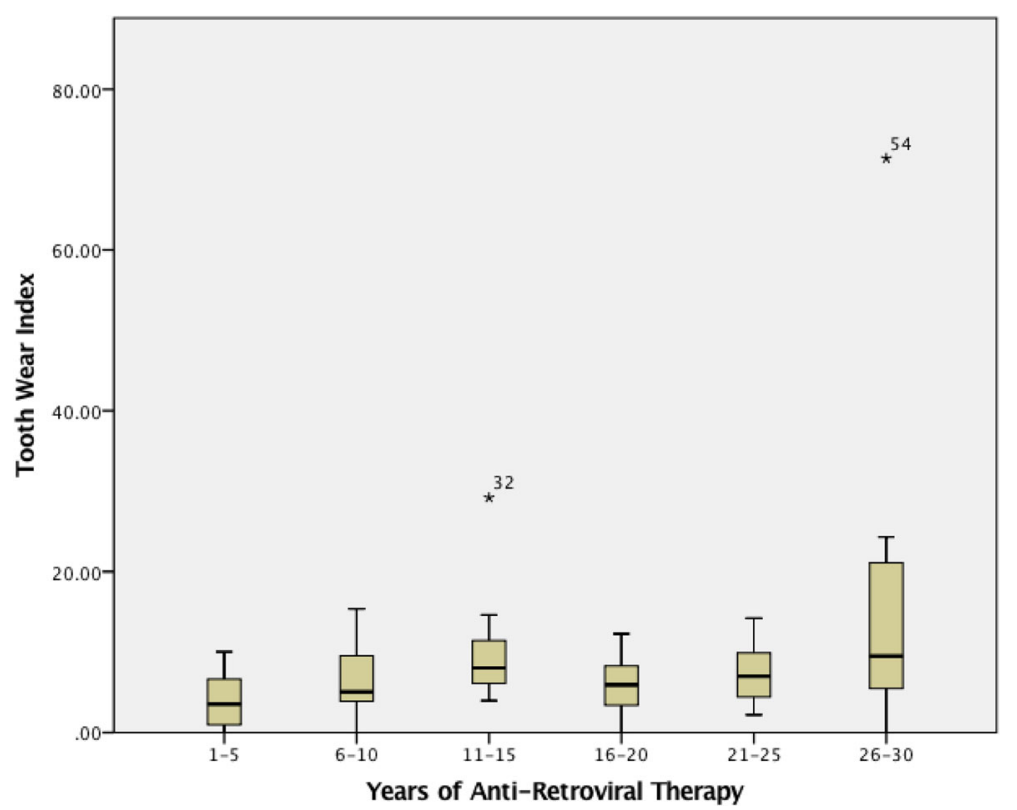

Fig. 1 Boxplot of Tooth Wear Index and Anti-Retroviral Therapy Duration $\left(n_{1-5 y r s}=8, n_{6-10 y r s}=14, n_{11-15 y r s}=10, n_{16-20 y r s}=12\right.$, $\mathrm{n}_{21-25 \mathrm{yrs}}=7, \mathrm{n}_{26-30 \mathrm{yrs}}=7$ )

After controlling for age, we observed that the years of anti-retroviral therapy use was positively correlated with tooth wear index $(\mathrm{B}=0.05$, $\mathrm{p}$-value $=0.076)($ Table 4$)$. The standardization of the tooth wear index as variables demonstrated that a five year increase in years of antiretroviral therapy was associated with a 0.154 standard deviation increase in tooth wear index after adjusting for age $(p=0.076)$. Due to the small sample size, this value may bear significance. However, this finding warrants further investigation in this area with a larger sample size. Notably, the standardized coefficient (beta) for years of retroviral therapy is greater than the standardized coefficient for age $(0.267$ vs. 0.142$)$. This

Table 4 Summary of Regression Analyses for Predicting Tooth Wear Index Among HIV + ve Individuals

\begin{tabular}{llllll}
\hline Variable (predictors) & \multicolumn{5}{l}{ Tooth Wear (Square Root) } \\
\cline { 2 - 6 } & $\mathrm{B}$ & Std. Error B & $\beta$ & $\mathrm{R}^{2}$ & $\mathrm{p}$ \\
\hline Age & 0.033 & 0.012 & 0.284 & 0.081 & $0.006^{* *}$ \\
Years of ART & 0.060 & 0.022 & 0.341 & 0.116 & $0.009^{* *}$ \\
Both Age and Years of ART & & & & 0.131 & $0.021^{*}$ \\
$\quad$ Years of ART & 0.047 & 0.026 & 0.267 & 0.076 \\
Age & 0.021 & 0.022 & 0.142 & 0.340 \\
HIV + ve & 0.780 & 0.322 & 0.246 & 0.061 & $0.017^{*}$ \\
Both HIV + ve and Age & & & & 0.131 & $0.002^{* *}$ \\
$\quad$ HIV + ve & 0.714 & 0.312 & 0.225 & & $0.025^{*}$ \\
$\quad$ Age & 0.031 & 0.011 & 0.266 & & $0.008^{* *}$ \\
\hline${ }^{*} p$-value< 0.05 & & & & & \\
${ }^{* *} p$-value 0.01 & & & & &
\end{tabular}

observation suggests that the years of antiretroviral therapy had a greater effect on tooth wear index than age.

"The transformation to square root of tooth wear index linearized the regression most effectively than tooth wear index, tooth wear index squared and log of tooth wear index. The log of tooth wear index excluded those with tooth wear indexes of 0 , thus we utilized the square root of tooth wear index transformation for data analysis and interpretation.

\section{Discussion}

Nearly $90 \%$ of HIV + ve subjects taking antiretroviral medications presented with tooth wear. The prevalence of tooth wear among HIV positive individuals found in our study is much greater than that reported by Juvino et al. [11]. This difference may be attributed to our study's use of the mean tooth wear index formula, whereas Juvino et al. diagnosed bruxism based on a selfreported questionnaire of bruxism behaviors. As suggested by Ekfeldt et al. [7], the tooth wear index formula our study utilized is a reliable indicator of bruxism. Our findings here suggest bruxism and the corresponding tooth wear is a greater problem in PWLHA than previously thought.

As supported by the literature [2,32], with nearly half of the HIV positive population presenting with a psychiatric disorder, there is a significantly greater prevalence of psychiatric disorders in PLWHA than non-infected subjects in our study. Many of the subjects reported 
taking psychiatric medications such as Celexa, Cymbalta, Lexapro and Ativan. These medications are used to treat depression and anxiety, which has been determined to be a common psychiatric disorder affecting this population $[2,32]$. Though we did not attempt to measure the proportion with anxiety and/or depression within HIV + ve and HIV-ve subject groups, our findings suggest this population is more affected by psychiatric disorders than non-infected individuals. Furthermore, those with HIV presented with both psychiatric disorders and tooth wear, which agrees with the findings of Piccoli et al. [23], who suggested that individuals with depression and anxiety have increased tooth wear.

Our study is the first to investigate the relationship between tooth wear and antiretroviral therapy. Our findings suggest that the longer an individual is on antiretroviral medication, the greater amount of tooth wear he or she will experience. This finding can help to explain the negative effects that antiretroviral medications, as well as psychiatric medications, can have on oral health [10, 24, 31, 33]. However, our findings cannot conclude with certainty that these medications have a direct effect on parafunctional oral habits. It remains challenging to conclude whether age was a major contributing factor to the amount of tooth wear we observed, as Bernhardt et al. [4] demonstrated that tooth wear is natural result of the aging process. We can draw from these findings that PLWHA clench and grind their teeth during both day and night in a much higher proportion and present with a mean tooth wear index about $50 \%$ more than those who do not have HIV. Another interesting finding was that the HIV + ve subjects were 95\% male. This may be yet another contributing factor to the greater mean tooth wear observed in the HIV + ve subject group. As Bernhardt et al. [4] as suggested, men are more likely to exhibit bruxist behavior, therefore experiencing greater amounts of tooth wear.

Similar to the study by Meless et al. [20], the findings from our study also suggest that knowledge of tooth wear plays a significant role in the comprehensive care of HIV-infected individuals. We must also consider the form of medication during treatment plan discussions for its age appropriateness. For instance, liquid medications often administered to children have cariogenic potential due to the acidity and sugar content $[24,31]$. These are a few of the many other factors medical providers must consider when establishing a comprehensive treatment plan for HIV patients to prevent unnecessary oral health problems.

All clinicians should be aware that SSRI antidepressants may cause bruxism or increase the intensity of symptoms. However, SSRIs are not the only medications linked to bruxism. Buspirone, an anxiolytic, is a receptor agonist that increases dopaminergic neuron firing in the ventral tegmental area and increases the synaptic release of dopamine in the prefrontal cortex [9]. These effects ameliorate drug-induced bruxism properties of SSRIs [9]. This finding may be the foundation for future studies on the treatments of these side effects. Importantly, because medical providers have been successful at treating the effects of SSRI-related bruxism [22], an interprofessional intervention between medical and dental providers may be the new way to approach a debilitating oral health care problem and improve health outcomes for PLWHA.

There are a few limitations to our study. Due to the small sample size, our findings cannot be generalized to all population groups. The findings of this study, however, do lay the foundation for further investigation on these populations. Because our sample was taken from a lower socioeconomic group in a community dental clinic, our sample of participants consists of individuals who are typically at a higher risk for developing dental problems, such as periodontal disease, caries, and enamel erosion. Though reliable, the tooth wear index formula is only one clinical measure of bruxism. There are various other intraoral devices that may be more accurate in assessing the degree of bruxism. Future research in this area can benefit from use of such devices.

\section{Conclusions}

The findings of our study demonstrate the importance of oral health side effects associated with the treatment of PLWHA. In patients with already compromised immune systems, chronic bruxism can have a significant impact on HIV positive patients' oral health and their quality of life. Although research in this area is in its beginning phase, our hope is to initiate a dialogue with medical and dental providers who care for HIV patients. The addition of a medical intervention may compliment and support successful prevention and treatment of bruxism. By educating providers about the potential harmful impact of HIV medications on patients' dentition and oral health, we can hopefully intervene with night guard therapy at the time a patient is diagnosed with HIV. By prescribing night guard therapy at the time of diagnosis, PLWHA patients will be better protected from the many negative effects associated with chronic bruxism, including temporomandibular disorder, headache, enamel erosion, and tooth wear. The integration of patients' systemic and oral health care needs will provide more comprehensive treatment for our patients and provide them with a higher quality of life.

\section{Acknowledgements}

We would like to thank Katrina Ramsey, MPH from the OHSU Biostatistics \& Design Program (partially supported by UL1TR002369 [OHSU CTSA]) for data analysis expertise. We are also thankful to all the participants of this study for their willingness to participate. 


\section{Authors' contributions}

RK, JA and GB designed the study. GB collected the data. HS, RK and EP analyzed the data and drafted the manuscript with input from all authors. All authors read and approved the final manuscript.

\section{Funding}

Russell Street Dental Clinic receives grant funding from United States Department of Health and Human Services (DHHS)'s Health Services Resources Administration's (HRSA) HIV/AIDS Bureau through different programs.

Oregon Clinical and Translational Research Institute (OCTRI) grant UL1TR002369 partially supported OHSU Biostatistics \& Design Program, which provided statistical support for this study.

\section{Availability of data and materials}

The datasets used and/or analyzed during the study are available from the corresponding author on reasonable request.

\section{Ethics approval and consent to participate}

This study protocol was reviewed and approved by the Oregon Health \& Science University Institutional Review Board (IRB00010706). A written informed consent was obtained from all study participants.

\section{Consent for publication}

Not applicable.

\section{Competing interests}

The authors declare they have no competing interest.

\section{Author details}

${ }^{1}$ Department of Periodontology, School of Dentistry, Oregon Health \& Science University, 5N034 SDPERI, 2730 SW Moody Ave, Portland, Oregon 97201-5042, USA. 'Department of Community Dentistry, School of Dentistry, Oregon Health \& Science University, Portland, OR, USA. ${ }^{3}$ School of Dentistry, Oregon Health \& Science University, Portland, OR, USA. ${ }^{4}$ Department of Endodontics, The University of Pennsylvania School of Dental Medicine, Philadelphia, PA, USA. ${ }^{5}$ Clinica Sierra Vista, Bakersfield, CA, USA.

\section{Received: 2 April 2019 Accepted: 7 June 2019}

Published online: 26 June 2019

\section{References}

1. American Academy of Family Physicians. (2011). Common Side Effects of HIV Medicines. American Family Physician, 83(12), 1456-1458.

2. American Psychiatric Association. (2008). Coping with AIDS and HIV. Dec 17 th.

3. Anttila S, Knuuttila M, Ylöstalo P, Joukamaa M. Symptoms of depression and anxiety in relation to dental health behavior and self-perceived dental treatment need. Eur J Oral Sci. 2006;114(2):109-14.

4. Bernhardt O, Gesch D, Splieth C, Schwahn C, Mack F, Kocher T, et al. Risk factors for high occlusal wear scores in a population-based sample: results of the study of health in Pomerania (SHIP). Int J Prosthodont. 2004;17:333-9.

5. Betancur MN, Lins L, Oliveira IR, Brites C. Quality of life, anxiety and depression in patients with HIV/AIDS who present poor adherence to antiretroviral therapy: a cross-sectional study in Salvador, Brazil. Braz J Infect Dis. 2017;21(5):507-14.

6. Chapple I, Hamburger J. The significance of oral health in HIV disease. Sex Transm Infect. 2000;76:236-43.

7. Ekfeldt A, Hugoson A, Bergendal T, Helkimo A. An individual tooth wear index and an analysis of factors correlated to incisal and occlusal wear in an adult Swedish population. Acta Odontol Scand. 1990;48:343-9.

8. Heslin KC, Cunningham WE, Marcus M, Coulter I, Freed J, Der-Martirosian C, et al. A comparison of unmet needs for dental and medical care among persons with HIV infection receiving care in the United States. J Public Health Dent. 2001;61:14-21.

9. Jaffee MS, Bostwick JM. Buspirone as an antidote to venlafaxine-induced bruxism. Psychosomatics. 2000;41:535-6.

10. Johansson AK, Omar R, Carlsson GE, Johansson A. Dental erosion and its growing importance in clinical practice: from past to present. International Journal of Dentistry. 2012;2012:632907.
11. Juvino AC, Pereira CB, Tini GF, Noro Filho GA, Mesquita AM, Giovani EM. Diagnosis of the prevalence of bruxism in HIV/AIDS patients, associated with psychological factors: pilot study. JSM Head and Face Medicine. 2017;2(1):1004.

12. Kampe T, Edman G, Bader G, Tagdae T, Karlsson S. Personality traits in a group of subjects with long-standing bruxing behaviour. J Oral Rehabil. 1997;24:588-93.

13. Koyano K, Tsukiyama Y, Ichiki R, Kuwata T. Assessment of bruxism in the clinic. J Oral Rehabil. 2008:35:495-508.

14. Lavigne GJ, Khoury S, Abe S, Yamaguchi T, Raphael K. Bruxism physiology and pathology: an overview for clinicians. J Oral Rehabil. 2008;35:476-94.

15. Luca M, Luca A, Grasso CM, Calandra C. Nothing to smile about. Neuropsychiatr Dis Treat. 2014;10:1999-2008.

16. Manfredini $D$, Landi N, Fantoni $F$, Segù M, Bosco M. Anxiety symptoms in clinically diagnosed bruxers. J Oral Rehabil. 2005;32(8):584-8.

17. Manfredini D, Lobbezoo F. Relationship between bruxism and temporomandibular disorders: a systematic review of literature from 1998 to 2008. Oral Surgery, Oral Medicine, Oral Pathology, Oral Radiology, and Endodontology. 2010;109:e26-50

18. Manfredini D, Winocur E, Guarda-Nardini L, Paesani D, Lobbezoo F. Epidemiology of bruxism in adults: a systematic review of the literature. J Orofac Pain. 2013;27(2):99-110.

19. Marcus M, Freed JR, Coulter ID, Der-Martirosian C, Cunningham W, Andersen $\mathrm{R}$, et al. Perceived unmet need for oral treatment among a national population of HIV-positive medical patients: social and clinical correlates. Am J Public Health. 2000;90:1059-63.

20. Meless D, Ba B, Faye M, Diby JS, N'zoré S, Datté S, et al. Oral lesions among HIV-infected children on antiretroviral treatment in West Africa. Tropical Med Int Health. 2014;19(3):246-55.

21. Menezes TO, Rodrigues MC, Nogueira BM, Menezes SA, Silva SH, Vallinoto AC. Oral and systemic manifestations in HIV-1 patients. Rev Soc Bras Med Trop. 2015;48(1):83-6.

22. Milanliglu A. Paroxetine-induced severe sleep bruxism successfully treated with buspirone. Clinics (Sao Paolo). 2012;67(2):191-2.

23. Piccoli L, Besharat LK, Cassetta M, Migliau G, Di Carlo S, Pompa G. Tooth wear among patients suffering from mental disorders. Annali di Stomatologia. 2014;5(2):52-60.

24. Pomarico L, Czauski G, Portela MB, de Souza IP, Kneipp L, de Araújo Soares RM, de Araújo Castro GF. Cariogenic and erosive potential of the medication used by HIV-infected children: $\mathrm{pH}$ and sugar concentration. Community Dent Health. 2008;25(3):170-2.

25. Preeja C, Ambili R, Nisha KJ, Seba A, Archana V. Unveiling the role of stress in periodontal etiopathogenesis: an evidence-based review. J Investig Clin Dent. 2013;4(2):78-83.

26. Rajan R, Sun YM. Reevaluating antidepressant selection in patients with bruxism and temporomandibular joint disorder. J Psychiatr Pract. 2017;23(3):173-9.

27. Schiødt M, Pindborg JJ. AIDS and the oral cavity: epidemiology and clinical oral manifestations of human immune deficiency virus infection: a review. Int J Oral Maxillofac Surg. 1987;16(1):1-14.

28. Schuster MA, Collins R, Cunningham WE, Morton SC, Zierler S, Wong M, et al. Perceived discrimination in clinical care in a nationally representative sample of HIV-infected adults receiving health care. J Gen Intern Med. 2005;20(9):807-13.

29. Sears B, Cooper C, Younai FS, Donohoe T. HIV discrimination in dental care: results of a discrimination testing study in Los Angeles County: The Williams Institute; 2011.

30. Shetty S, Pitti V, Satish Babu CL, Surendra Kumar GP, Deepthi BC. Bruxism: a literature review. Journal of Indian Prosthodontic Society. 2010;10(3):141-8.

31. Subramaniam P, Kumar K. Cariogenic potential of medications used in treatment of children with HIV infection. Special Care in Dentistry. 2014; 34(3):127-30.

32. Sun W, Wu M, Qu P, Lu C, Wang L. Psychological well-being of people living with HIV/AIDS under the new epidemic characteristics in China and the risk factors: a population-based study. Int J Infect Dis. 2014;28:147-52.

33. Younai FS, Marcus M, Freed JR, Coulter ID, Cunningham W, Der-Martirosian C, Guzman-Bercerra N, Shapiro M. Self-reported oral dryness and HIV disease in a national sample of patients receiving medical care. Oral Surgery, Oral Medicine, Oral Pathology, Oral Radiology, and Endodontology. 2001;92(6):629-36.

34. Zimpel RR, Fleck MP. Depression as a major impact on the quality of life of HIV-positive Brazilians. Psychology, Health \& Medicine. 2014;19(1):47-58.

\section{Publisher's Note}

Springer Nature remains neutral with regard to jurisdictional claims in published maps and institutional affiliations. 